\title{
Educación superior en contexto mapuche: El caso de La Araucanía, Chile ${ }^{1}$
}

\author{
Higher education in mapuche context: the case of La Araucanía, Chile
}

\section{Ensino superior no contexto mapuche: o caso de La Araucanía, Chile ${ }^{3}$}

Katerin Arias-Ortega

Universidad Católica de Temuco

Facultad de Ciencias de la Salud

Departamento de Psicología

Temuco, Chile

karias@uct.cl

(iD) http://orcid.org/0000-0001-8099-0670

Segundo Quintriqueo-Millán

Universidad Católica de Temuco

Facultad de Educación

Departamento Ciencias de la Educación

Temuco, Chile

squintri@uct.cl

(iD) http://orcid.org/0000-0002-7228-4095

Recibido • Received • Recebido: 26 / 09 / 2018

Corregido • Revised • Revisado: 30 / 01 / 2020

Aceptado • Accepted • Aprovado: 01 / 04 / 2020

\begin{abstract}
Resumen: El artículo analiza la educación superior en Chile en contexto mapuche, específicamente en La Araucanía. El método utilizado es un análisis y discusión bibliográfica que considera 4 libros de referencia, 17 artículos científicos y 8 documentos normativos, sobre educación superior en contexto indígena. La tesis plantea que la educación superior está arraigada a un racismo científico institucional que no incorpora la episteme indígena en la formación y construcción de conocimientos de estudiantes mapuches y no mapuches. Concluimos que el racismo científico en la educación superior trae consecuencias como bajos resultados académicos, debido a la descontextualización curricular. También, mantiene y perpetua las desigualdades sociales, académicas, políticas y económicas en el contexto regional, desvaloriza lo propio y valora lo ajeno.
\end{abstract}

Palabras claves: Educación superior; Epistemología pluralista; Interculturalidad; Racismo científico.

\footnotetext{
${ }^{1}$ Agradecimientos al proyecto Profondecyt 2019PF-KA-05 y Fondecyt 1181531.

${ }^{2}$ Thanks to the Profondecyt Project 2019PF-KA-05 and Fondecyt 1181531.

${ }^{3}$ Graças ao projeto Profondecyt 2019PF-KA-05 e ao Fondecyt 1181531.
} 
http://doi.org/10.15359/ree.24-2.1

ROR: https://ror.org/01t466c14 Universidad Nacional, Costa Rica

http://www.una.ac.cr/educare

educare@una.cr

\begin{abstract}
This article analyzes higher education in La Araucanía, a Mapuche region, Chile. The method used is an analysis and a bibliographic discussion that considers four reference books, 17 scientific articles, and eight normative documents on higher education in an indigenous context. The thesis suggests that higher education is rooted in institutional scientific racism that does not incorporate indigenous epistemology in the formation and construction of knowledge of Mapuche and non-Mapuche students. We conclude that scientific racism in higher education has brought consequences such as low academic results due to the decontextualization of curricula. This racism has also maintained and perpetuated social, educational, political, and economic inequalities in the regional context, devaluing what is proper and valuing what is foreign.
\end{abstract}

Keywords: Higher Education; Pluralist Epistemology; Interculturality; Scientific Racism.

Resumo: $\mathrm{O}$ artigo analisa o ensino superior no Chile em um contexto mapuche, especificamente em La Araucanía. O método utilizado é uma análise e discussão bibliográfica que considera 4 livros de referência, 17 artigos científicos e 8 documentos normativos sobre o Ensino Superior no contexto indígena. A tese sugere que a educação superior está enraizada em um racismo científico institucional que não incorpora a epistemologia indígena na formação e construção do conhecimento dos alunos mapuches e não mapuches. Concluímos que o racismo científico no ensino superior traz consequências, como a baixa escolaridade, devido à descontextualização dos currículos. Também mantém e perpetua as desigualdades sociais, acadêmicas, políticas e econômicas no contexto regional, desvalorizando o que é próprio e valorizando o que é estrangeiro.

Palavras-chaves: Educação superior; epistemologia pluralista; interculturalidade; racismo científico.

\title{
Introducción
}

La discusión bibliográfica que sostenemos sobre la educación escolar en contexto indígena, por ejemplo en Canadá, México, Ecuador, Colombia y Chile, nos permite constatar las siguientes problemáticas globales y particulares: 1) formación del profesorado descontextualizada al territorio y el conocimiento propio de estudiantes indígenas; 2 ) una baja cobertura curricular para estudiantes indígenas; 3) una desventaja socioeconómica entre indígena y no indígena, para acceder a la educación en simetría con estudiantes no indígenas; y 4) una dicotomía entre lo urbano y rural en cuanto a la cobertura y calidad de la educación en contexto indígena (Dietz y Mateos Cortés, 2011; Jerôme, 2013; León Correa, 2011; Ortelli y Sartorello, 2011). Estas problemáticas derivan en las siguientes consecuencias: 1) una brecha significativa en la escolarización entre indígenas y no indígenas; 2 ) omisión de los conocimientos y la lengua vernácula en la educación escolar; 3 ) reproducción del racismo científico institucionalizado, mediante la transmisión de contenidos disciplinarios del currículo escolar; 4) invisibilización de lo indígena en la educación escolar; y 5) el bajo éxito escolar y educativo de indígenas en comparación con no indígenas (Chávez, 2008; Cruz, 2016; Mansilla et ál., 2015; Quintriqueo et ál., 2015). El conjunto de estas problemáticas y sus consecuencias se constituyen en la construcción 
de una injusticia social y educativa hacia las poblaciones indígenas en general en las Américas. Esto justifica establecer una discusión teórica y empírica crítica sobre la situación de estudiantes indígenas en la educación superior, lo que permita sensibilizar a las instituciones y a quienes están a cargo de la gestión de la educación universitaria.

En lo específico, el presente artículo se relaciona con la incorporación de estudiantes indígenas en la educación superior en Chile, principalmente en contexto mapuche en La Araucanía. Esta región se caracteriza por la diversidad social y cultural, con diferentes lenguas indígenas y extranjeras de personas inmigrantes que cohabitan en un mismo espacio y que interactúan en la sociedad actual. En Chile, tanto en el contexto rural como urbano, habita un total de 1745147 personas que se autoidentifican como mapuches, equivalente a un 79,8\%, del total de población indígena a nivel nacional (2 185 792), de los cuales 321949 (34,3\%) vive en la región de La Araucanía (Ministerio de Desarrollo Social y Familia, Gobierno de Chile, 2017). De dicha población mapuche, la niñez y juventud de edad escolar asisten al sistema educativo en los niveles de Educación Parvularia, Educación Básica, Educación Media y Educación Superior.

En el contexto de la educación escolar chilena existe una verdadera brecha entre estudiantes, según sean estudiantes indígenas o no. Es así como en los estadísticos del Ministerio de Desarrollo Social y Familia, Gobierno de Chile (2017), se constata que en la población de 25 años o más, es común observar una diferencia de $2 \%$ y hasta $9 \%$ en relación con diferentes niveles educativos entre los grupos indígenas y no indígenas. En esta constatación, son los grupos de estudiantes indígenas quienes obtienen cada vez un menor nivel educacional. Este mismo tipo de brecha se observa entre la población urbana y rural, donde las tasas de analfabetismo en la población rural alcanzan el 5\%. En ese sentido, cuando se constata que en La Araucanía la población mapuche se concentra mayormente en el área rural, se observa que esta brecha es más evidente. Lo anterior se complementa con que la mayoría de población rural tiene como nivel educativo máximo alcanzado educación básica incompleta, bajo nivel de educación media completa o profesional y un bajo nivel de educación superior completo. Estas representaciones y datos, obtenidos del Ministerio de Desarrollo Social y Familia, Gobierno de Chile (2017), dan cuenta que la población mapuche en La Araucanía es la que obtiene menor nivel educativo en comparación con estudiantes no mapuches. La persistencia de esos bajos niveles educativos para las personas indígenas y rurales expresan las desigualdades estructurales en la sociedad. En este sentido, el sistema educativo escolar parece no ser capaz de ofrecer a los pueblos indígenas, las condiciones efectivas para finalizar con éxito los procesos de formación. De este modo, los programas generales de educación, no son pertinentes para que las personas indígenas superen las condiciones de vulnerabilidad.

Consideramos que estos bajos resultados educativos son, en alguna medida, una consecuencia de la situación social de pobreza en la que se encuentra La Araucanía. Dicha situación sienta sus bases en el período histórico de Pacificación de La Araucanía, proceso en el 
http://doi.org/10.15359/ree.24-2.1

ROR: https://ror.org/01t466c14 Universidad Nacional, Costa Rica

http://www.una.ac.cr/educare

educare@una.cr

cual se comienza con el empobrecimiento sistemático del pueblo mapuche por parte del Estado chileno. Este proceso se llevó a cabo mediante el despojo territorial de las personas mapuches, enajenándoseles más del noventa por ciento de su territorio histórico (Quintriqueo, et ál., 2015). Es así como a la población mapuche se les confina a pequeños espacios territoriales infértiles para la agricultura y ganadería de subsistencia, con lo cual se buscó condenarla a la pobreza económica. Esta realidad contrasta con la economía próspera del pueblo mapuche, anterior a la guerra de Pacificación, producto de la autodeterminación y control que tenía sobre su territorio (Quintriqueo et ál., 2015). Es así como en la actualidad, por ejemplo, La Araucanía es la región que históricamente ha ocupado el primer lugar en situación de pobreza en relación con el resto del país (Encuesta de Caracterización Socioeconómica Nacional, 2017). Actualmente, esta región obtiene el mayor porcentaje de pobreza por ingreso a nivel país, esto es equivalente a un $17,2 \%$. La pobreza por ingreso refiere a la situación de personas cuyo ingreso total mensual es inferior al mínimo establecido, es decir, \$ 264000 pesos chilenos. Esta carencia económica no permite satisfacer las necesidades básicas, alimentarias y no alimentarias, del grupo familiar (Ministerio de Desarrollo Social y Familia, Gobierno de Chile, 2017). Además, La Araucanía se caracteriza por ser la región que posee mayor pobreza multidimensional, alcanza un total de $25,4 \%$. La pobreza multidimensional refiere a la situación de personas indígenas y no indígenas que forman parte de hogares que no logran alcanzar condiciones adecuadas de vida, en relación a cinco dimensiones: 1) educación; 2) salud; 3) trabajo y seguridad social; 4) vivienda y entorno, y 5) redes y cohesión social (Ministerio de Desarrollo Social y Familia, Gobierno de Chile, 2017). Precisamos que estos niveles de pobreza persisten en La Araucanía, según los últimos resultados de la Encuesta de Caracterización Socioeconómica Nacional del 2017.

Desde la mirada del Estado, la situación actual en que se encuentra La Araucanía refiere a la carencia económica y categorización de vulnerabilidad. La vulnerabilidad es entendida como la situación de dependencia económica en que se encuentran grupos socialmente excluidos y minorizados, que pone en riesgo la autodeterminación y la libre elección de su cosmovisión y formas de enfrentar la vida (León Correa, 2011). Sin embargo, explicar los bajos resultados educativos obtenidos por la región implica considerar otros factores que no necesariamente son los económicos. De acuerdo con lo anterior, planteamos la siguiente tesis, en la cual sostenemos que, el profesorado y quienes están a cargo de la gestión educativa en escuelas situadas en contextos indígenas, especialmente en comunidades mapuches rurales, no tienen interés en el éxito escolar y educativo del estudiantado mapuche, campesino y de sectores sociales desfavorecidos. Esto se observa, por ejemplo, en la inadecuada gestión educativa en relación con procesos de enseñanza-aprendizaje descontextualizados (Arias-Ortega et ál., 2018), una baja cobertura curricular ofrecida y una sobrevaloración de la nota, principalmente en los niveles de Educación Básica y Educación Media. 
Lo anterior constituye una desventaja en relación con una alta cobertura curricular de los establecimientos escolares situados en centros urbanos centralizados, como es la ciudad de Temuco, donde la valoración de las notas se ajusta a la cobertura escolar (Arias-Ortega et ál., 2018). Además, en las escuelas situadas en comunidades rurales, no se consideran los conocimientos previos del estudiantado, así como sus características sociales y culturales, las cuales no necesariamente se condicen con el canon occidental de educación escolar (Quintriqueo et ál., 2015). Sostenemos que dichos elementos se constituyen en variables que podrían incidir negativamente en el éxito escolar y educacional de estudiantes en el sistema educativo escolar. El éxito escolar o académico, refiere a la adquisición y dominio de conocimientos, saberes y competencias, en coherencia y consistencia con los programas escolares y académicos formales. Permiten la sanción o reconocimiento formal expresada en la obtención de diplomas y certificados que formalizan la aprobación de un curso o nivel educativo; en tanto que el éxito educacional refiere al desarrollo global y óptimo de la persona, desde una perspectiva integral, que considera las dimensiones cognitiva, espiritual, afectiva, física y social de su ser (Perrenoud, 2007).

La metodología utilizada en este artículo consiste en un análisis y discusión bibliográfica, el que nos permite sistematizar antecedentes teóricos y empíricos, respecto de procesos educativos llevados a cabo en la educación superior por estudiantes indígenas en contextos interculturales. En la discusión se considera 4 libros de referencia, 17 artículos científicos o de divulgación y 8 documentos normativos, sobre educación superior en contexto indígena. Operacionalmente, se realiza una localización de artículos científicos, el que se operacionaliza de la siguiente manera: 1 ) se efectúa una revisión de artículos científicos en revistas nacionales e internacionales indexadas en Web of Science, Scopus, Scielo, ERIC, Latindex. Esto se realiza utilizando palabras clave, para la búsqueda de artículos científicos. Dichas palabras clave son: a) pueblos indígenas y educación superior; b) experiencias de estudiantes indígenas en la educación superior; c) educación superior en contexto indígena; y d) brechas educativas entre indígenas y no indígenas en la universidad. También se realizó una búsqueda de artículos o documentos normativos en relación con las mismas palabras clave. Los resultados de esta búsqueda arrojaron un total de 3390 artículos de revistas científicas, de divulgación científica y documentos normativos de nivel académico o gubernamental. De este total de artículos se escogieron 100 para su revisión, los cuales cumplían con los siguientes criterios de inclusión: 1) haber sido publicados en los últimos 10 años; y 2) artículos de divulgación de resultados de investigación sobre experiencias de estudiantes indígenas en la educación superior, publicados en Europa, Latinoamérica y Chile, en idioma inglés, francés, portugués y español. Este procedimiento nos permitió seleccionar 29 artículos que conforman el corpus de análisis central del presente artículo.

En esa perspectiva, el procedimiento de análisis de los artículos científicos, de carácter normativo y de divulgación científica se llevó a cabo a través de un análisis de contenido respecto 
http://doi.org/10.15359/ree.24-2.1

ROR: https://ror.org/01t466c14 Universidad Nacional, Costa Rica

http://www.una.ac.cr/educare

educare@una.cr

a las características, posibilidades y limitaciones que experimentan estudiantes indígenas en el sistema de educación superior. Es así como, a través del análisis de contenido, buscamos develar núcleos temáticos de orden abstracto que nos permitan aportar con una comprensión y explicación acerca de los componentes objetivos y subjetivos que inciden en la visibilización o invisibilización de estudiantes de ascendencia indígena en la educación superior. Asimismo, se buscó develar lineamientos pedagógicos y metodológicos, para abordar la inserción de estudiantes indígenas a la educación superior en contextos indígenas e interculturales. En efecto, esto nos permite contribuir con pistas de acción que promuevan la interculturalización de la educación superior, con base en prácticas educativas que incorporen una educación con pertinencia social y cultural, para comprender la realidad, desde una perspectiva local y global.

El objetivo del presente artículo es establecer una discusión teórica y empírica crítica sobre la situación de estudiantes indígenas en la educación superior, específicamente el caso de alumnado mapuche en La Araucanía, Chile.

\section{Presencia indígena en la educación escolar y universitaria}

Según datos del Ministerio de Educación de Chile (MINEDUC, 2016b), en el sistema educativo escolar nacional, en el año 2015 se registra una matrícula total de 3548736 estudiantes. Del total de estudiantes en el sistema educativo, 106.505 pertenecen a uno de los nueve pueblos indígenas reconocidos por el Estado chileno (Mapuche, Aymara, Likan Antai, Diaguita, Quechua, Colla, Rapanui, Yagan, Kawashkar), lo que representa un 3\%. Del total de 106505 estudiantes indígenas, un total de 89185 pertenecen al pueblo mapuche, esto equivale a un 83,7\% (MINEDUC, 2016b). De acuerdo con los datos, el pueblo mapuche tiene mayor representatividad de niñez y juventud insertas en los distintos niveles de educación escolar, matrícula que aumenta progresivamente en el tiempo. Es así como, al comparar estos datos, identificamos que la matrícula total de estudiantes a nivel nacional ha aumentado en 2 101, en el año 2016 llega a un total de 3550 837 en el año 2016. De este total 113756 personas se adscriben a algún pueblo indígena, donde observamos un aumento de 7251 estudiantes de ascendencia indígena. Esto significa que el aumento de la matrícula de estudiantes indígenas es mayor al aumento a nivel nacional. Esto podría significar una reidentificación progresiva de la población estudiantil de origen indígena en el país. De los 113756 estudiantes de ascendencia indígena, un total de 95075 pertenecen al pueblo mapuche, esto equivale a un 83,5\% (MINEDUC, 2016b).

En relación con la cantidad de estudiantes mapuches y resultados obtenidos en educación superior, en el contexto de La Araucanía, no existe información oficial. Sin embargo, algunas instituciones de manera particular han creado sus propios sistemas estadísticos, focalizándose solo en la identificación de estudiantes indígenas, sin considerar los logros académicos. Es así como en La Araucanía, mediante estadísticas internas de la Universidad Católica de Temuco 
http://doi.org/10.15359/ree.24-2.1

ROR: https://ror.org/01t466c14 Universidad Nacional, Costa Rica

(2017), se lleva un seguimiento de estudiantes de origen indígena que se han incorporado a la institución, durante los últimos siete años. Es lo que observamos en la Tabla 1, en la que se explicita la matrícula total por año académico y porcentaje de matrícula de estudiantes indígenas.

Tabla 1: Matrícula de estudiantes indígenas y no indígenas

\begin{tabular}{ccccc}
\hline Año académico & Matrícula total & $\begin{array}{c}\text { Matrícula estudiantes } \\
\text { indígenas }\end{array}$ & $\begin{array}{c}\text { Porcentaje de } \\
\text { representatividad }\end{array}$ & $\begin{array}{c}\text { Aumento de } \\
\text { matrícula }\end{array}$ \\
\hline 2012 & 7.082 & 1.459 & $20,6 \%$ & - \\
2013 & 6.692 & 1.609 & $20,92 \%$ & 150 \\
2014 & 8.334 & 1.980 & $23,77 \%$ & 371 \\
2015 & 8.680 & 2.240 & $25,81 \%$ & 260 \\
2016 & 10.003 & 2.762 & $27,61 \%$ & 522 \\
2017 & 10.542 & 3.023 & $28,68 \%$ & 241 \\
2018 & 10.646 & 3.119 & $30,34 \%$ & 96 \\
\hline
\end{tabular}

Nota: Elaboración propia en base a datos extraídos en el Sistema de Información Institucional KIMN (Universidad Católica de Temuco, 2017).

En la Tabla 1 se observa un aumento sistemático de la matrícula de ascendencia indígena en la UCT. Por ejemplo, en el año 2012 de un total de 7082 estudiantes, 1459 (20,6\%) son mapuches; en el 2017, de un total de 10 542, 3023 (28,68\%) son mapuches. Finalmente, en el año 2018 se observa que, de un total de 10646 estudiantes, 3119 son de ascendencia mapuche, equivalente a $30,34 \%$ de la matrícula. Sin embargo, aun cuando la matrícula de estudiantes mapuches ha aumentado, podemos observar que todavía es deficiente y baja en relación con el resto de estudiantes no mapuches que acceden a este nivel educativo. Esta es la realidad de una universidad regional, la que de manera interna ha establecido estadísticas. No obstante, la falta de precisión y medición del resto de universidades o el gobierno local no nos permite tener un panorama mucho mayor y preciso, respecto de la situación actual de La Araucanía. La falta de información podría estar ocultando datos y una realidad como lo es develar las disparidades académicas, sociales y el bajo acceso de estudiantes mapuches a la educación superior. O bien, invisibilizar el aumento progresivo de estudiantes mapuches a la educación superior, quienes potencialmente podrían incidir en explicitar y documentar demandas de política-reivindicación-autonomía-soberanía, en la relación entre academia y pueblos indígenas, los derechos a la educación de los pueblos indígenas, el parentescoterritorio de estudiantes y el conocimiento indígena. 
http://doi.org/10.15359/ree.24-2.1

ROR: https://ror.org/01t466c14 Universidad Nacional, Costa Rica

http://www.una.ac.cr/educare

educare@una.cr

Pensamos que la poca claridad y disponibilidad de información, respecto de estudiantes indígenas en educación superior, se debe a un racismo científico institucionalizado en este tipo de instituciones. El racismo científico institucionalizado es producto de la hegemonía del conocimiento de corte eurocéntrico occidental, que trae como consecuencia la invisibilización de los sujetos mapuches en las instituciones de educación superior. Además, la negación de sus saberes y conocimientos propios. Es así como el racismo científico institucionalizado se concibe desde la monoculturalidad eurocéntrico occidental, que posiciona el conocimiento disciplinar occidental por sobre el conocimiento indígena, al operar de manera invisibilizada la colonialidad del saber, del ser y del hacer en el marco de la institución escolar. Asimismo, la invisibilización del colonialismo en la educación escolar limita la posibilidad de incorporar la episteme del conocimiento indígena en los procesos de enseñanza y aprendizaje. Esto se acrecienta en la educación superior y dificulta una formación transdisciplinaria desde un enfoque educativo intercultural, para la formación de los futuros grupos de profesionales a nivel teórico, metodológico y empírico.

En el contexto chileno la educación superior se caracteriza por el amplio número de instituciones encargadas de impartir este nivel educativo, las cuales se inscriben en procesos de acreditación. La acreditación es un proceso voluntario al que se someten las instituciones de educación superior, para alcanzar una certificación de calidad en sus procesos internos (gestión institucional). Esto asegura el cumplimiento del proyecto de la institución, a través de mecanismos de autorregulación y aseguramiento de la calidad (CNED, MINEDUC, 2018). En el ámbito de las carreras de salud (medicina) y pedagogía la acreditación es obligatoria, según lo establece la Ley 20.129. Esta acreditación certifica la calidad de la educación en relación con los propósitos declarados y los criterios establecidos por las respectivas comunidades académicas y profesionales, para asegurar un alto nivel educativo en la formación de sus estudiantes.

Según datos del CNED, MINEDUC (2018), en Chile existen 47 centros de formación técnica, de los cuales 13 se encuentran acreditados y 34 aún no logran la acreditación. En relación con los institutos profesionales existen un total de 43, de los cuales 18 se encuentran acreditados y 25 aún no lo han conseguido. En Chile existen 27 universidades agrupadas en el Consejo de Rectores de Universidades chilenas (CRUCH), de las cuales solo 2 no se encuentran acreditadas. Además, existen 34 universidades privadas que no pertenecen al $\mathrm{CRUCH}$, de las cuales 22 están acreditadas al año 2018 y 12 aún no logran acreditarse. De acuerdo con los datos presentados, existe un total de 151 instituciones de educación superior, las cuales atienden en el año 2018 a un total de 1162306 estudiantes en pregrado a nivel nacional (CNED, MINEDUC, 2018).

Con base en los datos, observamos que La Araucanía en los años 2015-2016 ha tenido una variación considerable respecto de la cantidad de estudiantado matriculado en educación superior. En el año 2016 la región ascendió al tercer lugar a nivel país, fue una de las regiones con mayor matrícula en educación superior (MINEDUC, 2016a). No obstante, en la educación superior 
en general y en La Araucanía en particular, no existe una estadística abierta y disponible a la sociedad civil, respecto del porcentaje de estudiantado mapuche inserto en este nivel educativo.

Ahora bien, independiente de que el acceso a la educación superior presente un crecimiento considerable a nivel país, las características históricas de este sistema, aún no cambian (Moya, 2011). Por ejemplo, su configuración es altamente elitista y sustentada en modelos educativos traídos desde el extranjero como el enfoque por competencias (Turra et ál., 2015), el que no necesariamente responden al contexto social, político y económico de la región (Arias-Ortega et ál., 2018). En La Araucanía la educación superior se ha caracterizado por su composición altamente monocultural y monolingüe en castellano, se basa en un currículo único y centralizado para la formación de las personas profesionales. En este sentido, en los itinerarios formativos se prescinde de los saberes y conocimientos propios de los pueblos indígenas que componen el país. En el caso específico de La Araucanía, en la educación superior se prescinde de los saberes y conocimientos educativos propios, así como de las finalidades educativas del pueblo mapuche (Mansilla Sepúlveda et ál., 2015; Quintriqueo et ál., 2015). De este modo, la gestión educativa en educación superior, está sustentada en un enfoque educativo monocultural y funcional a las necesidades del Estado, y obvia las necesidades de la población que atienden y niega, con ello, una educación pertinente a la realidad social y cultural de La Araucanía. Producto de lo anterior, se invisibiliza la diversidad social y cultural, ya sea de lenguas indígenas y extranjeras, conocimiento indígena, local y campesino que portan los sujetos que conviven en un espacio territorial determinado (Mansilla Sepúlveda et ál., 2015; Tubino, 2014). Más aún, es un tipo de educación superior que busca la homogeneización social y cultural de los individuos que acceden a ella, perpetúa, de esta manera, una educación que aumenta la desigualdad social, la injusticia social y la segregación de los individuos pertenecientes a grupos minoritarios (Baudelot y Leclercq, 2008). Por ejemplo, pueblos indígenas, inmigrantes y personas que provienen del sector rural tienen igualdad de condiciones de acceso a la universidad, pero con instrumentos estandarizados que segregan a la población como lo es la Prueba de Selección Universitaria (PSU) (Vargas Moreno, 2014). Con ello, el sistema educativo en educación superior se constituye en un sistema ideado para discriminar y segregar la sociedad.

Desde un enfoque de gestión educativa monocultural, la educación superior en Chile perpetúa el racismo científico en la formación profesional en sus distintas disciplinas. Es decir, en ciencias básicas y en ciencias aplicadas, donde se niega la incorporación de cualquier otra forma de producción del conocimiento (Quintriqueo et ál., 2015). Lo anterior ha sido llevado a cabo mediante los siguientes mecanismos: 1) en Chile, la educación superior se ha concebido históricamente desde un pensamiento eurocéntrico occidental, desde la lógica de los grupos conquistadores, para construir en los sujetos un pensamiento único, para dar sentido y concebir el mundo (Arias-Ortega et ál., 2018); 2) en la educación superior solo es validado el conocimiento eurocéntrico occidental el que se constituye en la base de la sociedad para formar a la futura ciudadanía, según el orden social y moral establecido, lo que se explicita en los contenidos 
http://doi.org/10.15359/ree.24-2.1

ROR: https://ror.org/01t466c14 Universidad Nacional, Costa Rica

http://www.una.ac.cr/educare

educare@una.cr

disciplinarios transmitidos por el Estado nacional en el marco de la educación escolar (AriasOrtega et ál., 2018); 3) la educación superior se sustenta en un modelo por competencias, en el que se continúa ofreciendo una educación descontextualizada a la realidad local. Asimismo, se focaliza solo en el desarrollo académico desde lo disciplinario, por lo que está desarticulado de las experiencias de vida cotidiana de los sujetos en interacción con su medio local, regional, nacional, laboral, material y humano (Arias-Ortega et ál., 2018); 4) la educación superior prescinde de los saberes y conocimientos indígenas en la formación profesional, por lo cual, no considera las condiciones sociales y culturales en las que el futuro profesional se desempeñará.

De esta manera, la educación se transforma en una formación profesional descontextualizada que no incorpora en los itinerarios formativos los saberes y conocimientos locales, campesinos o de pueblos indígenas (Quintriqueo et ál., 2015; Tubino, 2014); y 5) las prácticas pedagógicas del profesorado son llevadas a cabo desde el monolingüismo en castellano y descontextualizadas del medio social y cultural (Arias-Ortega et ál., 2018). Es preciso señalar que estos mecanismos perpetúan el racismo científico en la educación superior como elementos, que ya se han naturalizado, por lo cual se han invisibilizado dentro de la misma universidad. Lo anterior es producto de una concepción universitaria desde la sociedad dominante, pensada solamente para universalizar el conocimiento eurocéntrico occidental. Pensamos que es necesario tomar consciencia que los sujetos que asisten a la educación superior son futuros profesionales que se desempeñarán en contextos indígenas e interculturales, por lo cual, ya no sirve el pensamiento monocultural como único y válido. Por ello, la necesidad de romper con esta lógica instrumental y técnica que caracteriza la educación superior como una institución desarraigada de los conocimientos existentes en su contexto regional y nacional.

\section{Experiencias de estudiantes indígenas en la educación superior}

Estudios recientes en el contexto latinoamericano han sistematizado experiencias educativas de estudiantes indígenas en la educación superior (Badillo Guzmán et ál., 2008; Bernasconi y Rojas, 2004; Chávez, 2008; Cruz, 2016; Ortelli y Sartorello, 2011; Reyes Pérez y López Santiago, 2015; Santana Colin, 2015) que dan cuenta de las tensiones y problemáticas en el medio universitario. Es así como los resultados de investigaciones de dichos autores encuentran un punto de convergencia, respecto a las tensiones que viven estudiantes indígenas en la educación superior. En efecto, se asume que el significado del ser indígena, en la universidad, implica visibilizar que el desarrollo de la vida universitaria no estará exento de conflictos interpersonales e intrapersonales, que se constituyen en conflictos interculturales. Según Ortorelli y Sartorello (2011), los conflictos interculturales surgen entre dos o más personas pertenecientes a sociedades y culturas diferentes, quienes poseen una base de conocimientos propios. Estos conflictos se fundamentan en relaciones prejuiciadas y estereotipadas hacia estudiantes indígenas o grupos minorizados socialmente. Pero al mismo tiempo, por una 
http://doi.org/10.15359/ree.24-2.1

ROR: https://ror.org/01t466c14 Universidad Nacional, Costa Rica http://www.una.ac.cr/educare

educare@una.cr

necesidad de reivindicación lingüística y cultural de estudiantes indígenas en el medio universitario, tanto en el ámbito de la disciplina de su formación profesional como en el ámbito de interacciones sociales y académicas.

En esa perspectiva, develamosquelas problemáticas que enfrenta el estudiantado indígena en la educación superior son: 1) problemas académicos asociados a los programas y beneficios que imparte el Estado hacia las personas indígenas, mediante políticas compensatorias y de discriminación positiva (Ortorelli y Sartorello, 2011; Reyes Pérez y López Santiago, 2015). De esta manera, se ofrece un sistema de financiamiento económico, para estudiantes indígenas que tienen un carácter reparatorio (Reyes Pérez y López Santiago, 2015). En relación con lo anterior, Santana Colin (2015) sostiene que las políticas compensatorias creadas para pueblos indígenas en educación superior han aumentado el acceso, pero no se asegura la permanencia en la universidad. Además, las becas no alcanzan a abarcar el costo total de matrícula, aranceles y manutención de estudiantes en los centros universitarios; 2 ) problemas personales de carácter socioafectivos refieren a la inseguridad de estudiantes indígenas y dificultades de adaptación en la educación superior. Esto, asociado a problemas de baja autoestima, timidez, soledad y falta de redes sociales (amigos y amigas), como características construidas por la educación escolar (Ortorelli y Sartorello, 2011; Reyes Pérez y López Santiago, 2015). Lo anterior incide en el logro de aprendizajes, en el desarrollo adecuado de la vida universitaria y, en ocasiones, trae consigo la deserción universitaria; 3) estudiantes mapuches poseen una lógica propia de educación familiar fundamentada en el kimeltuwün (acción educativa), que no siempre responde a la lógica de la educación universitaria (Quilaqueo Rapimán y Quintriqueo Millán, 2017). Es así como estudiantes indígenas se habitúan a luchar consciente o inconscientemente con un sistema educativo monocultural y monolingüe impuesto por la sociedad dominante, para desarrollar los procesos educativos, que no consideran la diversidad social, cultural y lingüística. Esto trae consigo, el desarrollo de una doble racionalidad de estudiantes indígenas, como una forma de pensar y ver la vida (Quilaqueo Rapimán y Quintriqueo Millán, 2017). Lo anterior le permite al estudiante indígena, enfrentar a un sistema fundado en patrones sociales y culturales impuestos por la sociedad y cultura hegemónica, que no necesariamente es acorde con sus propios marcos epistémicos (Reyes Pérez y López Santiago, 2015); y 4) problemas económicos, refiere a una ausencia de dinero relacionado con el lugar de procedencia de los estudiantes indígenas, considerando que la mayoría de ellos proviene de alguna comunidad rural, alejada de la zona urbana. Esto implica la necesidad de mayores recursos económicos, asociados a transporte y alimentación, así como para la compra de recursos educativos necesarios para enfrentar sus estudios universitarios. Por ejemplo, la compra de libros académicos, fotocopias, entre otros recursos para el aprendizaje (Chávez, 2008; Reyes Pérez y López Santiago, 2015). Desde esa perspectiva, pensamos que dichas tensiones y problemáticas deben ser consideradas en los itinerarios formativos y al momento de diseñar estrategias de enseñanza-aprendizaje en contextos indígenas e interculturales. Además, en la gestión administrativa de los programas de formación de pregrado. 
http://doi.org/10.15359/ree.24-2.1

ROR: https://ror.org/01t466c14 Universidad Nacional, Costa Rica

http://www.una.ac.cr/educare

educare@una.cr

En relación con lo anterior, un estudio desarrollado por Cruz (2016) devela que la educación superior en México, así como en otros países de Latinoamérica ha privilegiado una política monocultural y monolingüe en castellano que promueve la asimilación, inequidad lingüística y cultural. Esto evidencia la negación e invisibilización de un pluralismo epistemológico, plurilingüismo y pluriculturalismo que se constituyen en características propias de la sociedad actual en las Américas. Constatamos que el reconocimiento de la diversidad social y cultural se transforma en un asunto marginal y aislado en el proyecto de educación superior, lo cual se reflejaría en el arraigo de prácticas educativas de carácter monocultural. En esa perspectiva, la educación superior se transforma en el espejo de la sociedad actual, donde se observa una jerarquización sociocultural, discriminación y racismo científico hacia los grupos indígenas.

\section{Hacia una interculturalización de la educación superior}

Actualmente, la realidad educativa nos plantea el desafío de avanzar hacia una interculturalización de la educación superior, pertinente a su contexto local y regional, sin dejar de lado el aspecto global que permite a los sujetos movilizarse a otros contextos y en la frontera del conocimiento (Jérôme, 2013). Estos procesos de interculturalización involucran el desarrollo de capacidades, para relacionarse con sujetos pertenecientes a sociedades y culturas diferentes. Es así como el desarrollo e implementación de acciones y estrategias de formación para asegurar la calidad en educación en regiones es de vital importancia.

Desde esa visión, la gestión educativa para interculturalizar la educación superior implica contrarrestar la colonialidad del saber y del ser de profesoras y profesores formadores de formadores, quienes reproducen una formación colonial del futuro profesorado (Arias-Ortega et ál., 2018). Esto parte de que las universidades históricamente han sido creadas para universalizar el conocimiento y, por ende, la formación de personas, sustentada en el pretexto de la movilidad social y progreso de los individuos que allí asisten. Así, esta formación de carácter colonial instala en la mente de las futuras personas profesionales una concepción monocultural y eurocéntrica de la universidad. Esto implica enfrentar los retos para cubrir demandas en relación con la pertinencia de formar profesionales sensibles y con un alto compromiso social, ético y político con los pueblos indígenas, tanto en contexto regional, nacional como internacional (Rodríguez Varela et ál., 2015). De acuerdo con ello, diversos estudios (Gaete y Morales, 2011; Quintriqueo et ál., 2015; Rodríguez Varela et ál., 2015) plantean que el objetivo de mejorar el aprendizaje estudiantil, desde su realidad sociocultural es un aspecto central de la gestión educativa en educación superior. Esto, por las diversas demandas económicas, como lo es la globalización y competitividad de los mercados que exigen al sistema educativo una educación de calidad (Gaete y Morales, 2011). 
Otro reto de la interculturalización de la educación superior es superar la matriz del conocimiento 'mono epistémico' eurocéntrico occidental, para considerar la episteme indígena, articulada a distintas disciplinas, que en conjunto contribuirán en la construcción de conocimiento de frontera, desde un pluralismo epistemológico (Arias-Ortega et ál., 2018). Ello implica reconocer que no solo existe una sociedad del conocimiento, la cual debe permear el sistema educativo, sino más bien, reconocer las sociedades de conocimientos que evidencian el pluralismo epistemológico que compone la sociedad actual (Gaete y Morales, 2011). Sin embargo, en el caso de Chile, la gestión educativa ha estado centrada en una perspectiva epistemológica eurocéntrica occidental que valida y transmite un solo tipo de conocimiento 'científico', en desmedro de los saberes y conocimientos de la diversidad social y cultural que componen La Araucanía. Para ello, se debe partir por considerar que existen conflictos producto del racismo científico que niegan otras formas de producción del conocimiento (Quintriqueo et ál., 2015).

Desde allí se debe avanzar hacia una interculturalización que sea producto del diálogo inter-epistémico y la gestión educativa con pertinencia social y cultural en el medio universitario, para responder a las necesidades de las propias comunidades. Una posible estrategia es incorporar a la formación profesional, de manera articulada, los sistemas de conocimientos mapuche y occidental, desde su propio marco social y cultural de forma transversal y explicita en los itinerarios de la formación profesional (Vargas Moreno, 2014). Esto plantea la necesidad de transmitir la riqueza espiritual de la cosmovisión y epistemología indígena, tanto para futuros grupos profesionales indígenas como no indígenas (Ortorelli y Sartorello, 2011; Quilaqueo Rapimán y Quintriqueo Millán, 2017). Es así como la interculturalización de la educación superior implica proponer un conocimiento y pensamiento desde la pluralidad como multiplicidad de singularidades. Esto plantea el desafío de "concebir la [educación superior] como un espacio [donde] converge una multiplicidad de formas de pensar, conocer, concebir, imaginar y comprender la realidad educativa, en oposición al pensamiento colonial ... y universal" (AriasOrtega et ál., 2019, p. 10). Para ello, es necesario promover un giro decolonial, que surge no de la recuperación del pasado, sino de su reapropiación como pensamiento crítico (Quilaqueo Rapimán y Quintriqueo Millán, 2017).

En contexto mapuche, la educación superior presenta el desafío de abrirse a otras formas de enseñar y aprender, al incorporar la subjetividad y la espiritualidad que está a la base de la educación familiar, que permita cuestionar la hegemonía y monoculturalidad del conocimiento científico occidental. Desde esta perspectiva, en la medida que se avance en un diálogo de saberes entre los sujetos que pertenecen a distintas sociedades y culturas, podríamos avanzar en un proyecto de universidad intercultural que implique una co-construcción de conocimientos, desde un pluralismo epistemológico (Jérôme, 2013). Eso implica asumir la responsabilidad de generar acciones que promuevan la incorporación de 'otros saberes' en la formación 
http://doi.org/10.15359/ree.24-2.1

ROR: https://ror.org/01t466c14 Universidad Nacional, Costa Rica

http://www.una.ac.cr/educare

educare@una.cr

profesional, en el ámbito de distintas disciplinas como en la investigación, para deconstruir la ciencia occidental considerada como la única forma válida de construir, producir y reproducir conocimiento. Sostenemos que solo así se puede avanzar en la construcción del conocimiento y no desde las respuestas de orden colonial y monocultural eurocéntrico, sino más bien, desde una propuesta para construir un saber en conjunto, que busque el bienestar del individuo y del medio comunitario. En consecuencia, la interculturalización de la educación superior debiese trascender los límites y la capacidad del modelo occidental y traducir los saberes de la dimensión social y cultural de los pueblos indígenas como conocimientos válidos para la formación profesional de todo el estudiantado.

En efecto, interculturalizar la educación superior significa implementar nuevas formas de pensar la formación de las futuras personas profesionales para contextos indígenas e interculturales, donde las verdades absolutas, saberes y conocimientos educativos únicos sean cuestionados, desde otras visiones, desde otras formas de descubrir y construir el conocimiento (Quilaqueo Rapimán y Quintriqueo Millán, 2017). Implica definir otras fronteras del conocimiento en el ámbito académico, epistémico, curricular, para demarcar otras formas de concebir la enseñanza y el aprendizaje. En consecuencia, pensar en la interculturalización de la universidad es posicionarse en una forma de construcción, comprensión y explicación de la realidad, con el otro ser. Por ello, sostenemos que no basta con aumentar la incorporación de estudiantes indígenas a la educación superior. También implica, acompañar este proceso mediante la incorporación simétrica de los saberes y métodos educativos indígenas en los procesos de formación profesional de todo el estudiantado, desde su validez ontológica. Junto con ello, implica la participación real de las comunidades de los territorios desde colaboración mutua, para definir las ofertas y servicios educativos, teniendo en consideración un desarrollo profesional que responda a las necesidades e intereses del contexto local y regional.

En síntesis, sostenemos que las principales limitaciones prácticas de interculturalizar la educación superior son las siguientes: 1) no basta solo con incorporar contenidos educativos indígenas en los programas de formación, sino que es necesario que esos conocimientos tengan una simetría epistémica con el conocimiento occidental. Esto, ya sea en el momento de realizar estudios, practicas progresivas como en prácticas profesionales con la implicación del medio comunitario; 2) revertir el racismo y discriminación en personal académico, estudiantes y en quienes lideran la gestión universitaria; 3) concretizar prácticas de formación universitaria con la implicación de familias y comunidades indígenas; 4) formalizar la intervención de sabios indígenas en la formación de los grupos de futuros profesionales; 5) cambio de estructura organizacional y del conocimiento disciplinario que está a la base de la formación profesional; y 6) co-construir un enfoque educativo intercultural con actores del medio educativo, universitario, comunidades indígenas y sociedad civil, que sustenten el desarrollo de una interculturalización de la educación superior. 


\section{Discusión y conclusiones}

La revisión de la bibliografía nos ha permitido constatar que la educación superior, en su estado actual, da cuenta de una escasa presencia de estudiantes indígenas en su nivel educativo. Asimismo, evidenciamos que los planes y programas de estudio de las universidades, generalmente, no incorporan conocimientos indígenas de forma explícita al currículo de formación profesional. Aunque en algunas universidades existen experiencias aisladas de incorporación de contenidos educativos indígenas, que han surgido de manera autónoma, como iniciativas y buenas intenciones de una parte del personal académico y estudiantes dentro de las universidades. Es lo que observamos, por ejemplo, en las Universidades del Consejo de Rectores de Chile y en algunas universidades privadas, que dictan diplomados, curso de lengua indígena, cursos de artesanía, dirigidos a estudiantes y personal académico del área de ciencias sociales y humanas. Sin embargo, estas experiencias se constituyen en situaciones aisladas, no perdurables en el tiempo y no forman parte de la política institucional universitaria, ya sea por la falta de apoyo formal o por la carencia de recursos económicos en las universidades, para promover prácticas pedagógicas desde perspectivas interculturales.

En ese contexto, sostenemos que el sistema de educación superior continúa cristalizando la idea de asimilación cultural de estudiantes indígenas al sistema monocultural imperante en los niveles educativos del sistema educativo chileno. Precisamos que esta realidad es similar en algunos países caracterizados por una historia de colonización como es México, donde la presencia de estudiantes indígenas en la educación superior es baja en relación a la población no indígena. Asimismo, la educación que se entrega al estudiantado tiene características similares a la de Chile sustentada en la homogeneización cultural (Dietz y Mateos Cortés, 2011). Además, se constata que la baja cobertura de educación superior para los indígenas se suma a la falta de movilidad hacia la elite científica y académica en las universidades. No obstante, las normativas legales vigentes en Chile como la Ley Indígena, 19253 (Gobierno de Chile, Ministerio de Desarrollo Social, 1993) y el Convenio de la OIT 169 (Organización Internacional del Trabajo, 1989) demandan la accesibilidad y la movilidad de la población indígena en todos los niveles educativos y fundamentados en el conocimiento propio de estos pueblos. Así, las universidades no representan aún un medio para el desarrollo pleno de la cultura e historia de los pueblos indígenas en sus planteles educativos, tanto en Chile, como en La Araucanía en particular.

En ese contexto, los Estados promueven una educación superior que aún se encuentra en la etapa de políticas compensatorias o focalizadas solo en la incorporación de mayor cantidad de estudiantes indígenas a sus planteles educativos, pero no procura entregarles una educación pertinente a su contexto local. Un estudio de Turra et ál. (2015), sobre profesorado novel que egresa de carreras de educación diferencial, respecto a cómo está preparado para su desarrollo profesional muestra resultados de investigación dan cuenta de que el profesorado nuevo declara no estar preparado para desarrollarse en contextos diversos social y culturalmente (Turra et ál., 2015). 
http://doi.org/10.15359/ree.24-2.1

ROR: https://ror.org/01t466c14 Universidad Nacional, Costa Rica

http://www.una.ac.cr/educare

educare@una.cr

Para revertir dicha problemática, la Universidad Católica de Temuco declara en su política institucional el componente intercultural en los últimos 10 años, para la formación de sus futuros grupos profesionales. Esta política la distingue por visibilizar el aumento progresivo de estudiantes indígenas en su plantel educativo. Sin embargo, los lineamientos y formas de entender esta es difusa, en sus distintas facultades y sus departamentos. Entonces, el profesorado, personal académico y el propio estudiantado no encuentran una visión clara para el establecimiento de acciones que permitan responder a los sellos y finalidades educativas que se declaran en la universidad. Por ejemplo, a pesar de que aproximadamente un tercio de sus estudiantes pertenecen a la población mapuche, no se observan unidades de servicios y representación de dicha población en los estamentos universitarios. Esto podría ser la causa por la que no existe mayor información y explicación sobre la deserción, el éxito académico y educativo de estudiantes mapuches en la universidad.

En esa perspectiva, concluimos que las principales causas de exclusión del mapuche en la educación superior son: 1) la imposición de la educación escolar y universitaria de carácter monocultural eurocéntrico occidental para los pueblos indígenas; 2) la diferencia epistémica para concebir y explicar la pedagogía y educación, lo que genera conflictos epistemológicos, para comprender la formación profesional; 3) el sistema educativo escolar chileno está diseñado y se implementa para construir una segregación social, cultural y económica entre indígenas y no indígenas; 4) la lógica hegemónica que construye una educación solo para el sector indígena; y 5) la educación escolar monocultural eurocéntrica occidental que limita la movilidad educativa, social y económica para el grupo indígena.

Las principales pistas de acción que proponemos para revertir la situación de exclusión de estudiantes mapuches en la educación superior son: 1) un sistema educativo universitario centrado en lograr el aprendizaje de estudiantes indígenas y no indígenas, desde su marco social y cultural propio, en articulación con el conocimiento occidental; 2) enmarcarse en la construcción de un proyecto de educación universitaria que considere la episteme mapuche para contribuir a la producción del conocimiento a nivel regional y nacional como base para la interculturalización de la educación superior, para contrarrestar progresivamente el racismo científico arraigado en las instituciones de educación escolar; 3) implica una política de educación universitaria que permita crear nuevos espacios de cuestionamiento epistemológico, pedagógico, institucional y metodológico, desde una perspectiva intercultural. Consideramos, que pensar esta interculturalización desde un enfoque educativo intercultural implicaría a la familia y al medio comunitario; 4) promover la participación familiar y comunitaria de los pueblos indígenas en el diseño, aplicación y ejecución de programas educativos, que incorporen los conocimientos educativos mapuches al itinerario formativo de manera transversal en todas las carreras universitarias; 5) implementar espacios de participación, diálogo y acciones de indagación que promuevan la co-construcción de aprendizajes entre sujetos que pertenecen a 
sociedades y culturas diferentes. Esto nos permitiría avanzar en la de-colonialidad de prácticas pedagógicas, para transformar las formas tradicionales y estáticas de los procesos de enseñanzaaprendizaje. De este modo, las universidades situadas en contextos indígenas e interculturales enfrentan el desafío de incorporar la episteme indígena para enriquecer la formación profesional y la gestión universitaria, para co-construir un enfoque educativo intercultural, constituyéndose en agentes de desarrollo social, político, económico y educacional tanto para indígenas como no indígenas en La Araucanía.

\section{Referencias}

Arias-Ortega, K., Quilaqueo, D. y Quintriqueo, S. (2019). Educación intercultural bilingüe en La Araucanía: Principales limitaciones epistemológicas. Educação e Pesquisa, 45, e192645, 1-16. https://dx.doi.org/10.1590/s1678-4634201945192645

Arias-Ortega, K., Quintriqueo, S. y Valdebenito, V. (2018). Monoculturalidad en las prácticas pedagógicas en la formación inicial docente en La Araucanía, Chile. Educação e Pesquisa, 44, e164545, 1-19. https://doi.org/10.1590/s1678-4634201711164545

Badillo Guzmán, J., Casillas, M. y Ortiz Méndez, V. (2008). Políticas de atención a los grupos indígenas en la educación superior mexicana: El caso de la universidad veracruzana. Cuadernos Interculturales, 6(10), 33-61. http://www.redalyc.org/articulo.oa?id=55261004

Baudelot, C. y Leclercq, F. (2008). Los efectos de la educación. Del estante.

Bernasconi, A. y Rojas, F. (2004). Informe sobre la educación superior en Chile: 1980-2003. Editorial Universitaria Buchner.

Chávez Arellano, M. E. (2008). Ser indígena en la educación superior: ¿Desventajas reales o asignadas? Revista de la Educación Superior, 37(148), 31-55. http://www.scielo.org.mx/ scielo.php?script=sci arttext\&pid=S0185-27602008000400003\&lng=es\&tIng=es

Consejo Nacional de Educación, Ministerio de Educación Chile. (2018). Sistema de Información de Educación Superior del Consejo Nacional de Educación. https://www.cned.cl/indices/ matricula-institucionesprogramas-educacion-superior

Cruz, G. (2016). Discursivos reflexivos de estudiantes indígenas en universidades públicas de cuño tradicional de Oaxaca, México. Calidad en la Educación, 44, 243-274 https://doi. org/10.4067/S0718-45652016000100009

Dietz, G. y Mateos Cortés, L. S. (2011). Interculturalidad y educación intercultural en México: Un análisis de los discursos nacionales e internacionales en su impacto en los modelos educativos mexicanos. SEP. 
http://doi.org/10.15359/ree.24-2.1

ROR: https://ror.org/01t466c14 Universidad Nacional, Costa Rica

http://www.una.ac.cr/educare

educare@una.cr

Gaete, M. y Morales, R. (2011). Articulación del sistema de educación superior en Chile: Posibilidades, tensiones y desafíos. Calidad en la Educación, 35, 51-89. https://doi. org/10.4067/S0718-45652011000200003

Gobierno de Chile, Ministerio de Desarrollo Social. (28 de setiembre de 1993). Ley núm. 19253 por la que se establecen normas sobre protección, fomento y desarrollo de los indígenas y se crea la Corporación Nacional de Desarrollo Indígena (CONADI).

Jérôme, L. (2013). La coconstruction des connaissances en milieu muséal: Le Musée de la civilisation et sa nouvelle exposition permanente sur et avec les Premières Nations et les Inuit du Québec. En C. Juin (Ed.), Cahiers Dialog (pp. 25-29).

León Correa, F.J. (2011). Pobreza, vulnerabilidad y calidad de vida en América Latina: Retos para la bioética. Acta bioethica, 17(1), 19-29. https://doi.org/10.4067/S1726-569X2011000100003

Mansilla Sepulveda, J., Becerra Pena, S y Merino, M. E. (2015). Curriculum violence: Occidental knowledge hegemony in relation to indigenous knowledge. Procedia - Social and Behavioral Sciences, 190, 434-439. https://doi.org/10.1016/j.sbspro.2015.05.022

Ministerio de Desarrollo Social y Familia, Gobierno de Chile. (2017). Encuesta de caracterización socioeconómica de Chile CASEN. Ampliando la mirada sobre la pobreza y la desigualdad. Diagnóstico nacional y principales resultados regionales. Editorial Ministerio de Desarrollo y Planificación.

Ministerio de Educación de Chile. (2016a). Consejo Nacional de Educación. Centro de Estudios, División de Planificación y Presupuesto.

Ministerio de Educación de Chile. (2016b). Estadísticas de la educación. Centro de Estudios, División de Planificación y Presupuesto.

Moya, C. (2011). Equidad en el acceso a la educación superior: Los "cupos de equidad" en la Facultad de Ciencias Sociales de la Universidad de Chile. Calidad en la Educación, 35, 255275. https://scielo.conicyt.cl/pdf/caledu/n35/art11.pdf https://doi.org/10.31619/caledu. $\underline{\mathrm{n} 35.104}$

Organización Internacional del Trabajo. (1989). Convenio 169 sobre pueblos indígenas y tribales en países independientes. Ministerio de desarrollo Social, CONADI.

Ortelli, P. y Sartorello, S. C. (2011). Jóvenes universitarios y conflicto intercultural. Estudiantes indígenas y mestizos en San Cristóbal de Las Casas, Chiapas. Perfiles Educativos, 33 (Especial), 115-128. http://www.scielo.org.mx/scielo.php?script=sci abstract\&pid=S0185$\underline{26982011000500011 \& \operatorname{lng}=e s \& n r m=i s o}$ 
Perrenoud, P. (2007). Pedagogía diferenciada: De las intenciones a la acción. Editorial popular.

Quilaqueo Rapimán, D. y Quintriqueo Millán, S. (2017). Métodos educativos mapuches: Retos de la doble racionalidad educativa. Ediciones Universidad Católica de Temuco.

Quintriqueo, S., Quilaqueo, D., Peña-Cortés, F. y Muñoz, G. (2015). Conocimientos culturales como contenidos de la educación familiar mapuche. ALPHA, 40, 131-146. https://doi. org/10.4067/S0718-22012015000100010

Reyes Pérez, O de J. y López Santiago, N. (2015). Problemas y desafíos en la educación superior: El caso de los estudiantes indígenas de la región Costa del Estado de Oxaca. Temas de Ciencia y Tecnología, 56, 39-48.

Rodríguez Varela, D., De León Lafuente, L. A. y Galarza López, J. (2015). Los retos actuales de las instituciones de educación superior en el área de la gestión. Revista Universidad y Sociedad, 7(2), 52-55. http://scielo.sld.cu/pdf/rus/v7n2/rus07215.pdf

Santana Colin, Y. (2015). Educación superior de carácter intercultural para los pueblos indígenas en América Latina: Experiencias, tensiones y retos. Revista Colombiana de Educación, 69, 97-119. https://doi.org/10.17227/01203916.69rce97.119

Tubino, F. (2014). El transfondo epistémico de los conflictos interculturales. Revista Contextos Latinoamericanos, 11, 1-5. http://www.revistascientificas.udg.mx/index.php/CL/issue/ view/285/showToc

Turra, O., Valdebenito, V. y Torres, A. (2015). Teaching competencies for performing in school vulnerability contexts in Chile. Procedia - Social and Behavioral Sciences, 197, 1236-1241. https://doi.org/10.1016/j.sbspro.2015.07.386

Universidad Católica de Temuco. (2017). KIMN Sistema de Información Institucional. http://kimn2. uct.cl/

Vargas Moreno, P. A. (2014). Educación superior intercultural en disputa. Trayectorias de la Universidad Intercultural Amawtay Wasi. Polis, Revista Latinoamericana, 13(38), 269-300. https://doi.org/10.4067/S0718-65682014000200013 